\title{
The Frequency Modulation Response of Vertical-Cavity Surface-Emitting Lasers: Experiment and Theory
}

\author{
Andreas Hangauer, Member, IEEE, Jia Chen, Member, IEEE, Rainer Strzoda, \\ and Markus-Christian Amann, Fellow, IEEE
}

\begin{abstract}
The FM response of vertical-cavity surface-emitting lasers (VCSELs), i.e., the dynamic wavelength tuning behavior, is scrutinized. The FM amplitude and phase shift are measured up to $80 \mathrm{MHz}$ for GaAs-, InP-, and GaSb-based VCSELs from 763 to $2300 \mathrm{~nm}$. From measurements, it is found that the FM response consists of three components: intrinsic thermal tuning (dominating to several megahertz) with characteristic $1 / \sqrt{i f}$ behavior, the plasma effect (dominating from several megahertz), and a small effect (10-100 Hz) caused by the interaction of laser chip and submount. All effects are modeled and the measurement data are fitted to obtain effective thermal diffusivities, strength of the plasma effect, and time constant of the laser chip submount interaction. Comparing thermal models with different asymptotic behaviors, an approximation of the heat source in the laser with a nonzero thickness turned out to be necessary. Due to the plasma effect, with influence starting at $100 \mathrm{kHz}$, VCSELs cannot be considered a minimum phase system, which makes separate amplitude and phase measurements essential for device characterization. The " $N$ time constants model" is the proper choice for empirical description of the intrinsic thermal tuning component. The best fit coefficients to a rational frequency response are given for use in time-domain simulation programs.
\end{abstract}

Index Terms-FM phase shift, FM response, Hilbert transform, plasma effect, thermal time constant, thermal tuning, verticalcavity surface-emitting laser (VCSEL).

$\mathbf{K}$ NOWLEDGE of the dynamics of the laser tuning coefficient is important in all applications where tunable lasers are employed. In tunable diode laser spectroscopy, the laser is tuned to record absorption spectra and knowledge of the wavelength response at specific current modulation of the used tunable lasers is very important. Since the wavelength tuning is predominantly a thermal effect and heat conduction is a "slow" process, the wavelength response does not instantaneously follow the modulation current. The small signal dynamics are fully described by the FM response or the frequency-dependent cur-

Manuscript received December 1, 2010; revised January 22, 2011; accepted January 24, 2011. Date of publication March 22, 2011; date of current version December 7, 2011.

A. Hangauer and J. Chen are with the Walter Schottky Institute, Technical University of Munich, Garching 85748, Germany and also with Corporate Research and Technologies, Siemens AG, Munich 80200, Germany (e-mail: andreas.hangauer@wsi.tum.de; jia.chen@wsi.tum.de).

R. Strzoda is with the Corporate Research and Technologies, Siemens AG, Munich 80200, Germany (e-mail: rainer.strzoda@siemens.com).

M.-C. Amann is with the Walter Schottky Institute, Technical University of Munich, Garching 85748, Germany (e-mail: mcamann@wsi.tum.de).

Color versions of one or more of the figures in this paper are available online at http://ieeexplore.ieee.org.

Digital Object Identifier 10.1109/JSTQE.2011.2110640 rent tuning coefficient. There the amplitude and phase of the wavelength response to sinusoidal current modulation is specified versus frequency (for mathematical definition see Appendix A). The wavelength response to arbitrary current modulation waveforms can then be computed with a Fourier transform.

In the majority of the literature, the intrinsic thermal behavior of lasers has been modeled as first-order low-pass [1]-[5]. This is, however, not in agreement with experimental data both for vertical-cavity surface-emitting lasers (VCSELs) and DFB lasers [6], [7]. Analytical and physical models suited for the specific geometry of DFB lasers are given in [8, Sec. 4.7] [9]. Other work treats this issue by simply fitting a model with two or more time constants to the experimental data [10], [11]; however, the determined parameters cannot be related to physical quantities.

In contrast to DFB lasers, VCSELs have advantages such as low-power consumption, on wafer testability, and low-cost potential. Hence, these are very attractive light sources for different applications. In the literature, few work relates to the dynamic tuning behavior of VCSELs. The theory in [12] is expected to be inexact at high frequencies due to the assumption of an infinitely thin heat source and light mode. Therefore, in this paper this model is extended to fit the FM response of different VCSELs in a broader frequency range. It is based on two universal approaches and its aim is modeling for understanding of the device behavior and computer simulation. The relevant measurement data presented here were partly published before in [13] and [14].

\section{MEASURED FM RESPONSE AND ITS CHARACTERISTIC COMPONENTS}

In Fig. 1, measurement data for a 2.3- $\mu \mathrm{m}$ VCSEL [15], [16] are shown. At frequencies of several megahertz, a constant tuning coefficient is observed and a phase shift of $-180^{\circ}$ is approached. Between the cutoff starting at $\sim 10 \mathrm{kHz}$ and this constant region, a behavior $1 / f^{n}$ with $n$ around 0.5 is observed in the magnitude response (visible as slope $-1 / 2$ in the double logarithmic scale). At low frequencies (see the insets in Fig. 1), a small but characteristic dip in the phase shift and a small step in the tuning coefficient response are found. It is a small effect but was found to be present in all examined VCSELs in this study. A model for the FM response for VCSELs that accounts for these three effects (summarized in Table I) is developed in the next section. 


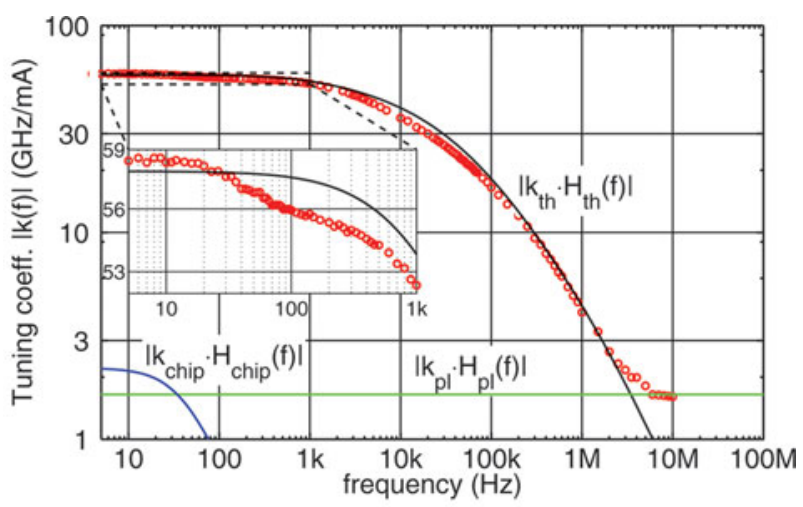

(a)

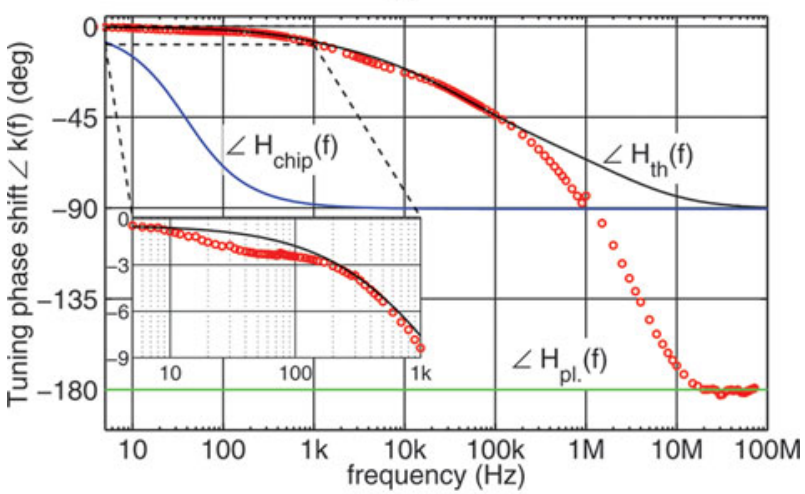

(b)

Fig. 1. (a) Amplitude and (b) phase of the tuning coefficient for a $2.3-\mu \mathrm{m}$ VCSEL (circles). The individual additive contributions from the intrinsic thermal tuning $H_{\mathrm{th}}$ (black), interaction between laser chip and submount $H_{\text {chip }}$ (blue), and plasma effect $H_{\mathrm{pl}}$ (green) inside the laser are shown as solid lines. (a) Tuning coefficient amplitude. (b) Tuning phase shift.

TABLE I

OVERVIEW OF THE CHARACTERISTIC COMPONENTS OBSERVED IN VCSEL FM RESPONSES (SEE FIG. 1)

\begin{tabular}{|l|l|}
\hline $\begin{array}{l}\text { Amplitude constant at }>1-5 \mathrm{MHz}, \text { phase reaches }-180^{\circ} \\
\text { ("plasma effect", } H_{p l} \text { ) }\end{array}$ & see II-B \\
\hline $\begin{array}{l}\text { Amplitude behavior is } f^{-n}(n \approx 0.5) \text { between cutoff and } \\
\text { const region ("intrinsic thermal tuning", } H_{t h} \text { ) }\end{array}$ & see II-A \\
\hline $\begin{array}{l}\text { Additional step in amplitude and peak in phase response } \\
\text { around } 100 \mathrm{~Hz} \text { ("laser chip-submount interaction", } H_{\text {chip }} \text { ) }\end{array}$ & see II-C \\
\hline
\end{tabular}

\section{ANALYSIS AND MODEL OF THE FM RESPONSE}

From the measurement, it can be concluded that the frequency-dependent tuning coefficient $k(f)$ is a superposition of three contributions

$$
k(f)=k_{\mathrm{th}} H_{\mathrm{th}}(f)+k_{\mathrm{pl}} H_{\mathrm{pl}}(f)+k_{\mathrm{chip}} H_{\text {chip }}(f)
$$

with coefficients $k_{\mathrm{th}}, k_{\mathrm{pl}}$, and $k_{\mathrm{chip}}$ modeling contributions from the intrinsic thermal tuning, the plasma effect, and the interaction between laser chip and the submount. The normalized functions $H_{\mathrm{th}}(0)=H_{\mathrm{pl}}(0)=H_{\text {chip }}(0)=1$ model the respective frequency dependence. For all examined VCSELs, typical values are in the range $k_{\mathrm{th}} \approx k(0), k_{\mathrm{pl}} \approx-0.02 \ldots-0.1 k(0)$, and $k_{\text {chip }} \approx 0.03 k(0)$. The $3-\mathrm{dB}$ frequencies for $H_{\text {th }}$ are in the several kilohertz to $100 \mathrm{kHz}$ range, for $H_{\mathrm{pl}}$. in the $1-20 \mathrm{GHz}$ range, and for $H_{\text {chip }}$ around $5-100 \mathrm{~Hz}$.

\section{A. Intrinsic Thermal Tuning}

The current tuning behavior is dominantly a thermal effect at low frequencies. It is caused by the temperature dependence of the effective optical length (geometric length times' effective refractive index) of the cavity resulting in an increasing wavelength with temperature. The dominant contribution comes from the refractive index increase with temperature. The thermal expansion of the cavity only contributes approximately $10 \%$ of the overall thermal wavelength tuning [17, Sec. 3.2.3].

The first-order low-pass model is unsuited for the intrinsic thermal model because it does not reproduce the slope of $n \approx 0.5$ above the thermal cutoff. The analytic VCSEL FM response model [12] is better suited because it reaches an asymptotic slope of $-1 / 2(1 / \sqrt{i f}$, "square root behavior"). Its phase shift reaches only $-45^{\circ}$ that does not allow for the combined model (thermal and plasma effect) to reach the high phase shift that is practically observed. The model [12] is based on the assumption of an infinitely thin heat source and mode distribution. In Appendix C, it is explained that if a plane or line heat source has a nonzero thickness $h$, a transition from square root behavior $(1 / \sqrt{i f})$ to $1 /($ if $)$ behavior will occur at a frequency given by approximately $\kappa /\left(\pi h^{2}\right)$ with $\kappa$ being the thermal diffusivity. It is clear that in a real device the heat source has some thickness even if it is expected to be very thin in VCSELs. Then the modeled FM response can both reproduce the slope of $-1 / 2$ after the cutoff and an asymptotic slope of -1 with a $-90^{\circ}$ phase shift. The "transition frequency" from slope $-1 / 2$ to slope -1 is adjusted by the thickness of the heat source and light mode. The refined model is based on the following approximations.

1) The material inside the laser is homogeneous but nonisotropic, i.e., has different thermal conductivities in $r$ and $z$ directions.

2) The heat is generated in the active region with radius $R_{Q}$ and is radially Gaussian distributed. Its thickness is $Z_{Q}$ with Gaussian distribution also in longitudinal direction. The heat source distribution $Q(x, y, z)$ is given by

$$
Q(x, y, z)=\frac{1}{(2 \pi)^{3 / 2} R_{Q}^{2} Z_{Q}} e^{-\frac{x^{2}}{2 R_{Q}^{2}}-\frac{y^{2}}{2 R_{Q}^{2}}-\frac{z^{2}}{2 Z_{Q}^{2}}} .
$$

3) The wavelength is determined by the average temperature in the laser (average with respect to mode distribution). The light mode $M(x, y, z)$ is laterally and longitudinally Gaussian distributed with radius $R_{M}$ and thickness $Z_{M}$ :

$$
M(x, y, z)=\frac{1}{(2 \pi)^{3 / 2} R_{M}^{2} Z_{M}} e^{-\frac{x^{2}}{2 R_{M}^{2}}-\frac{y^{2}}{2 R_{M}^{2}}-\frac{z^{2}}{2 Z_{M}^{2}}}
$$

4) The substrate, located at distance $D$ below the active region, is kept at constant temperature

$$
T(x, y,-D)=0 .
$$

The laser model including the approximations is illustrated in Fig. 2. The model in [12] is then contained as a special case with $Z_{M}=Z_{Q} \rightarrow 0$. In Appendix $\mathrm{B}$, it is shown that for the solution only the combined radii or thicknesses of heat source and light mode are relevant. The impulse response of the intrinsic thermal tuning behavior $h_{\mathrm{th}}(t)$ is proportional to the evolution 


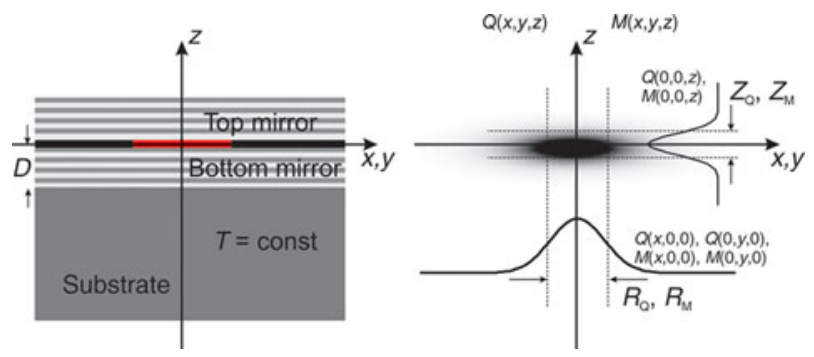

Fig. 2. Schematic of the laser model (left) and the heat source $Q$ and mode distribution $M$ inside the laser (right). Both $Q$ and $M$ are assumed to be Gaussian but with different radii $\left(R_{Q}, R_{M}\right)$ and thicknesses $\left(Z_{Q}, Z_{M}\right)$. The distance of the active region (at the coordinate origin) to the substrate (assumed to be an ideal heatsink) is $D$.

of the average temperature over time in response to a temporal heat pulse of unit strength (see Appendix B):

$$
h_{\mathrm{th}}(t) \propto \frac{1}{\sqrt{t+\frac{1}{2 \pi f_{Z}}}\left(t+\frac{1}{2 \pi f_{R}}\right)}\left(1-\exp \left(-\frac{1 /\left(2 \pi f_{D}\right)}{t+\frac{1}{2 \pi f_{z}}}\right)\right)
$$

with characteristic frequencies that directly relate to distances in the laser

$$
f_{R}=\frac{\eta_{R} \kappa_{\mathrm{bulk}}}{\pi\left(R_{Q}^{2}+R_{M}^{2}\right)}, \quad f_{Z}=\frac{\eta_{Z} \kappa_{\mathrm{bulk}}}{\pi\left(Z_{Q}^{2}+Z_{M}^{2}\right)}, \quad f_{D}=\frac{\eta_{D} \kappa_{\mathrm{bulk}}}{2 \pi D^{2}}
$$

were $\eta_{R} \kappa_{\mathrm{bulk}}, \eta_{Z} \kappa_{\mathrm{bulk}}$, and $\eta_{D} \kappa_{\mathrm{bulk}}$ are the relevant thermal diffusivities of the active region material in lateral direction, longitudinal direction, and the "effective" diffusivity of the material between active region and heat sink, respectively. Since an average diffusivity is difficult, if not impossible, to obtain a priori, the $\eta$ values are used as fit parameters. The bulk diffusivity $\kappa_{\text {bulk }}$ depends on the laser material system and is given by $0.31 \mathrm{~cm}^{2} / \mathrm{s}$ for GaAs, $0.372 \mathrm{~cm}^{2} / \mathrm{s}$ for InP, and $0.23 \mathrm{~cm}^{2} / \mathrm{s}$ for GaSb. The $\eta$ values are typically much smaller than 1 because of several effects.

1) For the ternary or quaternary material the laser contains, the thermal conductivity (and so the diffusivity) can drop by one to two orders of magnitude compared to the bulk value (binary material).

2) In layered structures such as a distributed Bragg reflector (DBR), the multitude of interfaces can cause the diffusivity in growth direction to drop to about $30 \%$ of the bulk value [18].

3) Uncertainties in the width or thickness of the light mode or heat source distribution.

The FM response is given as the Fourier transform of (5):

$$
H_{\mathrm{th}}(f) \propto \int_{0}^{\infty} \frac{1-\exp \left(-\frac{1 /\left(2 \pi f_{D}\right)}{t+\frac{1}{2 \pi f_{z}}}\right)}{\sqrt{t+\frac{1}{2 \pi f_{Z}}}\left(t+\frac{1}{2 \pi f_{R}}\right)} e^{-2 \pi i f t} \mathrm{~d} t .
$$

Note that the proportionality constant is chosen in such a way that $H_{\mathrm{th}}(0)=1$. Note that a closed-form expression for (7) only exists in the case $f_{Z} \rightarrow \infty\left(Z_{Q}=Z_{M} \rightarrow 0\right)$ [12, eq. (8) with $f_{0}=f_{R}$ and $d=\sqrt{f_{R} / f_{D}}$. Efficient numerical evaluation of (7), where the desired frequency points are distributed over several orders of magnitude, can be carried out in the manner explained in Appendix D.

\section{B. Plasma Effect}

The plasma effect is the dominant effect that causes a dependence of the refractive index on the carrier density in lasers [8, Sec. 4.5]. Since the carrier density in the active region is very high, even a small relative modulation of the carrier density will also cause a laser wavelength modulation. Compared to thermal tuning, the tuning by the plasma effect is broadband with cutoff frequencies in the gigahertz range and acts inversely to thermal tuning (phase $-180^{\circ}$ ). More precisely, the tuning coefficient contribution caused by the plasma effect is described by the laser rate equations. The linearized rate equations can be solved if a spatially homogeneous laser model is assumed. According to [5, Sec. 5.2], we get

$$
k_{\mathrm{pl}}=\frac{\alpha_{H}}{4 \pi e} \frac{\partial G}{\partial S} \quad H_{\mathrm{pl}}(f)=\frac{1+i f / f_{g}}{1+i f / f_{d}-f^{2} / f_{r}^{2}}
$$

with $\alpha_{H}$ the linewidth enhancement factor [19] and $\partial G / \partial S$ the dependence of the normalized gain on photon number $S$. It models the gain dependence on light intensity, which can be caused by several physical effects [20]. The characteristic frequencies $f_{r}$ (relaxation frequency), $f_{d}$ (damping frequency), $f_{g}$ typically lie in the gigahertz range [5]. For frequencies, $f<$ $100 \mathrm{MHz} H_{\mathrm{pl}}$ is essentially flat so in this paper $H_{\mathrm{pl}} \equiv 1$ is assumed (see Fig. 1, green curve). Note that spatial effects like spatial hole burning in VCSELs may cause a low-frequency roll-off that is not described by (8) [21].

\section{Laser Chip-Submount Interaction}

The additional small contribution at low frequencies is due to interaction of the laser chip and the submount. This is presented and modeled in this paper for the first time. All investigated lasers were packaged in a commercial TO5 housing including a thermo-electric cooler (TEC). In this setup, the laser chip was placed on an insulating $\mathrm{Al}_{2} \mathrm{O}_{3}$ submount which is the reason for the observed effect.

From measured data, it is evident that a small process is present that accounts to $2-4 \%$ of the overall thermal tuning and has cutoff frequencies in the $10-100 \mathrm{~Hz}$ range (also see Fig. 5, blue line). This explains the additional weak step in the tuning coefficient amplitude in Fig. 1(a) and the small peak in the tuning phase shift in Fig. 1(b) (at $f<200 \mathrm{~Hz}$ ). With finite element method computer simulations that include the laser chip and the submount, it was possible to reproduce this effect. Simulations without the submount, where the laser chip is placed on a constant temperature body, did not show this effect. It can be explained as follows: when the laser current is modulated, also the dissipated electric power is modulated. The heat is essentially removed through the submount and so also small temperature variations on the heatsink of the laser chip are created. This additional thermal resistance together with the heat capacity of the submount and the laser chip then cause this effect to have a cutoff frequency in the $10-100 \mathrm{~Hz}$ range. Although the exact physical description of this process would include many 
parameters it is a weak effect and high accuracy modeling is not required. Therefore it is modeled by a simple first-order low-pass with a single time constant (or cutoff frequency $f_{\text {chip }}$ ), i.e.,

$$
H_{\text {chip }}(f)=\frac{1}{1+i f / f_{\text {chip }}} \text {. }
$$

\section{Impossibility OF ReCONSTRUCTION OF THE FM Phase FROM FM AMPLITUDE}

It is well known that real and imaginary parts of the frequency response of a causal filter are the Hilbert transform of each other. Practically, all physical systems are, thus, fully described by the imaginary or real part of the frequency response only and a measurement of either will be sufficient for full characterization. However, one may also ask if a similar relationship holds for the amplitude and phase shift of the frequency response. This is possible if the system fulfills additionally to causality the minimum phase condition. Then, the Kramers-Kronig relations hold for amplitude and phase. According to systems theory of time discrete systems, the system

$$
\log H(f)=\log A(f)+i \phi(f)
$$

is causal if and only if $H(f)=A(f) e^{i \phi(f)}$ is a causal and minimum phase system [22]. Thus, the log amplitude response $\log A(f)$ and $\phi(f)$ are a Hilbert transform pair

$$
\phi(f)=-\frac{1}{\pi} \int_{-\infty}^{\infty} \frac{\log A(\nu)}{f-\nu} \mathrm{d} \nu .
$$

For proper convergence, it is essential to use the Cauchy principle value integral and to integrate also over the negative part of the spectrum. Since $\log A(f)$ is symmetric for real-valued systems, (11) is equivalent to the Kramers-Kronig relation

$$
\phi(f)=-\frac{2 f}{\pi} \int_{0}^{\infty} \frac{\log A(\nu)}{f^{2}-\nu^{2}} \mathrm{~d} \nu .
$$

From (11), it follows that a minimum phase system with asymptotic slopes of $0,-1 / 2$, and -1 in a double logarithmic plot of $A(f)$ has asymptotic phase shifts of $0^{\circ},-45^{\circ},-90^{\circ}$, respectively. A slope of $-n$ corresponds to $A(f) \rightarrow 1 / f^{n}$ behavior for $f \rightarrow \infty$.

The minimum phase reconstruction for the measurement of the $2.3 \mu \mathrm{m}$ VCSEL was computed using the method described in Appendix D and is shown in Fig. 3. An excellent agreement between measured phase and reconstructed phase at low frequencies is observed, which indicates that the thermal tuning component alone (which is dominating at low frequencies) is a minimum phase system. Deviations start at $f>100 \mathrm{kHz}$ and show that the presence of the plasma effect (which dominates at high frequencies) causes the laser tuning behavior to be a nonminimum phase system. In a minimum phase system, the observed constant tuning coefficient at several megahertz should cause an associated $0^{\circ}$ phase shift, which is different from the observed $-180^{\circ}$ phase shift. This can also be seen in Fig. 1(b) where the phase shift of the intrinsic thermal component $H_{\text {th }}$ starts to deviate at around $100 \mathrm{kHz}$ from the measurement. Remarkably, the influence of the plasma effect is stronger in the FM
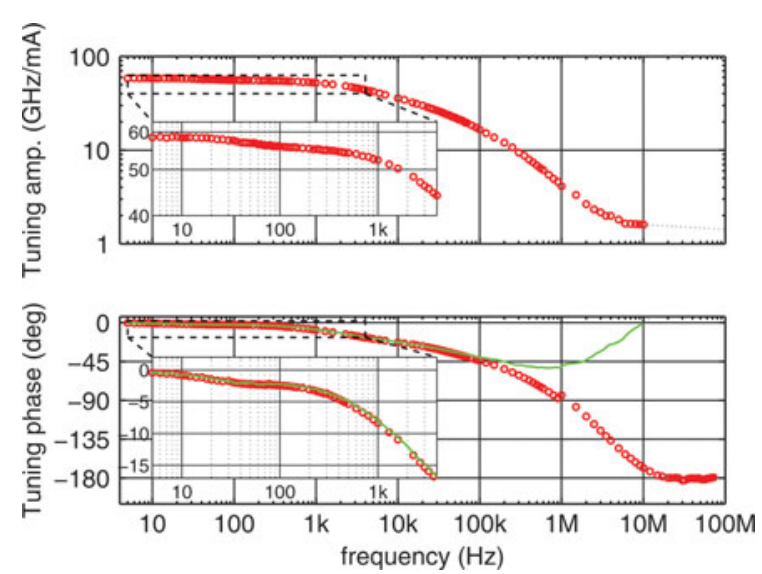

Fig. 3. Measured tuning coefficient amplitude (circles, top) and tuning phase shift (circles, bottom) for an InP-based 2.3- $\mu$ m VCSEL as well as the minimum phase reconstruction from amplitude using the Hilbert transform (11) (solid line, bottom). The agreement for low frequencies is excellent, proving the consistency between amplitude and phase measurement. At higher frequencies $(f>100 \mathrm{kHz}$ ) deviations are observed due to the stronger influence of the plasma effect, which essentially destroys the minimum phase property of the tuning behavior.

TABLE II

INVESTIGATED VCSELS AND THEIR CHARACTERISTIC PARAMETERS

\begin{tabular}{|l||l|l|l|l|}
\hline Wavelength & $763 \mathrm{~nm}$ & $1854 \mathrm{~nm}$ & $2365 \mathrm{~nm}$ & $2330 \mathrm{~nm}$ \\
\hline Substrate & GaAs & InP & InP & GaSb \\
\hline Top Mirror & $\begin{array}{l}\text { Epitactical } \\
\text { DBR }\end{array}$ & $\begin{array}{l}\text { Epitactical } \\
\text { DBR }\end{array}$ & $\begin{array}{l}\text { Epitactical } \\
\text { DBR }\end{array}$ & $\begin{array}{l}\text { Dielectric } \\
\text { DBR }\end{array}$ \\
\hline $\begin{array}{l}\text { Bottom Mir- } \\
\text { ror }\end{array}$ & $\begin{array}{l}\text { Epitactical } \\
\text { DBR }\end{array}$ & $\begin{array}{l}\text { Dielectric } \\
\text { DBR }\end{array}$ & $\begin{array}{l}\text { Dielectric } \\
\text { DBR }\end{array}$ & $\begin{array}{l}\text { Epitactical } \\
\text { DBR }\end{array}$ \\
\hline Aperture & $\begin{array}{l}\text { Lateral } \\
\text { Oxidation }\end{array}$ & $\begin{array}{l}\text { Buried Tun- } \\
\text { nel Junction }\end{array}$ & $\begin{array}{l}\text { Buried Tun- } \\
\text { nel Junction }\end{array}$ & $\begin{array}{l}\text { Buried Tun- } \\
\text { nel Junction }\end{array}$ \\
\hline Heatsink & $\begin{array}{l}\text { GaAs Sub- } \\
\text { strate }\end{array}$ & Gold & Gold & $\begin{array}{l}\text { GaSb Sub- } \\
\text { strate }\end{array}$ \\
\hline Reference & {$[23]$} & {$[24]$} & {$[15],[16]$} & {$[25],[26]$} \\
\hline $\begin{array}{l}R_{Q},\left(R_{M}\right. \\
\left.0.6 R_{Q}\right)^{4}\end{array}$ & $1.5 \mu \mathrm{m}^{1}$ & $2.5 \mu \mathrm{m}^{1}$ & $3.25 \mu \mathrm{m}^{1}$ & $2.5 \mu \mathrm{m}^{1}$ \\
\hline$D$ & $\approx 4 \mu \mathrm{m}^{2}$ & $\approx 2.4 \mu \mathrm{m}^{2}$ & $2.58 \mu \mathrm{m}^{2}$ & $8.18 \mu \mathrm{m}^{2}$ \\
\hline$Z_{Q}, Z_{M}{ }^{4}$ & $1.5 \mu \mathrm{m}^{3}$ & $0.42 \mu \mathrm{m}^{3}$ & $0.68 \mu \mathrm{m}^{3}$ & $0.68 \mu \mathrm{m}^{3}$ \\
\hline
\end{tabular}

${ }^{1}$ Current aperture radius. Light mode radius assumed to be $60 \%$ of aperture.

${ }^{2}$ Thickness of the bottom mirror and layers between mirror and active region.

${ }^{3}$ Penetration depth of the light mode into one mirror plus the thicknesses of additional layers between the mirror and the active region.

${ }^{4}$ Entries multiplied by $\sqrt{2 \log 2} \approx 1.18$ for conversion of a "standard deviation" to a "HWHM (half-width as half-maximum)".

phase shift because there deviations are more pronounced than in the amplitude response. The fact that the laser tuning behavior is no minimum phase system shows that both tuning phase shift and amplitude measurements are required for proper device characterization and correct prediction of the wavelength response for arbitrary current modulation waveforms. For the other investigated VCSELs, a very similar behavior is obtained: the plasma-effect influences the FM phase shift also starting at around $100 \mathrm{kHz}$.

\section{MEAsurement And Fit Results}

All measured devices are single-mode and continuous-wave laser devices, which were placed on a ceramic submount on top of a TEC for temperature stabilization. Operation temperature was slightly above room temperature. An overview of the devices and their characteristic parameters is given in Table II. 


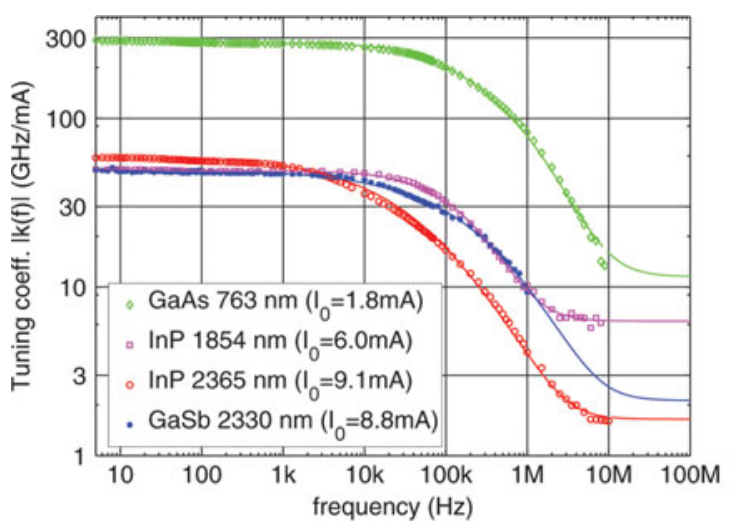

(a)

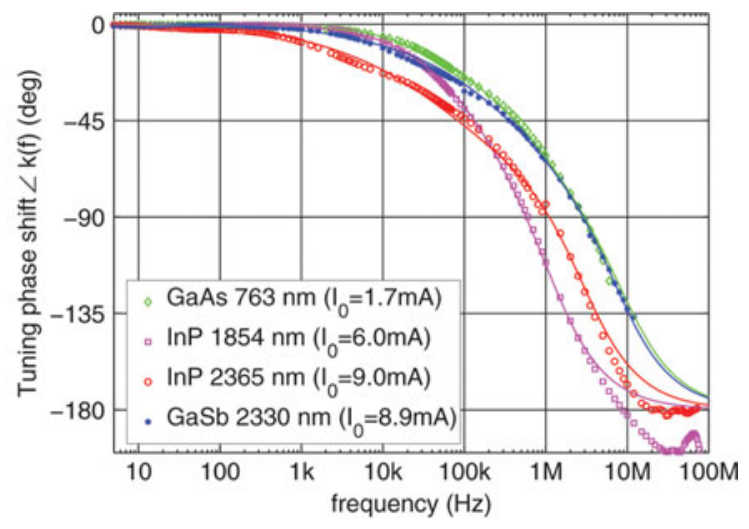

(b)

Fig. 4. (a) Absolute value and (b) phase of the tuning coefficient $k(f)$ versus frequency for measurement (markers) and fit to theoretical model (1) with (7) (solid lines).

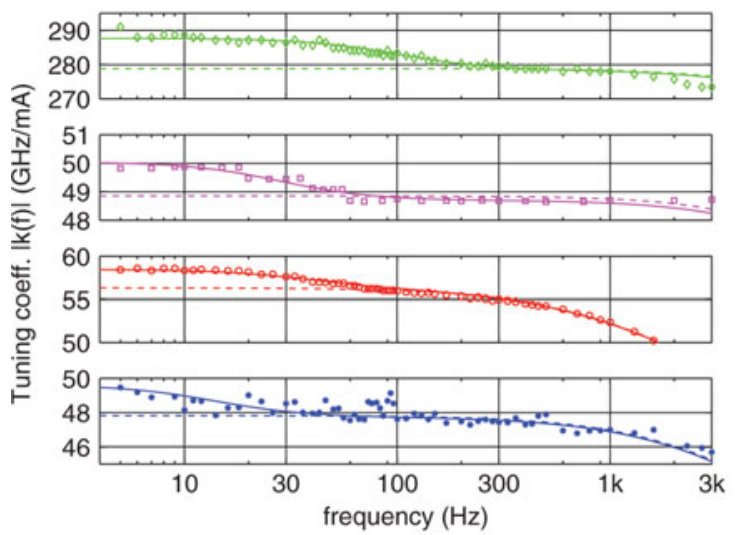

(a)

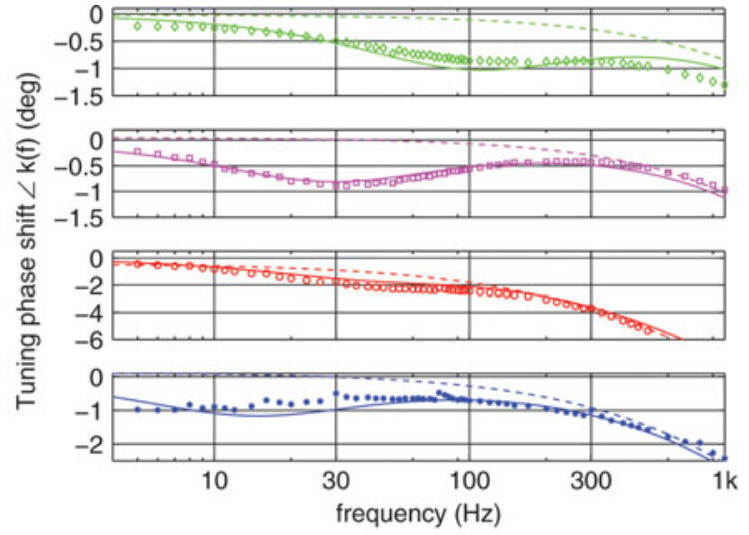

(b)

Fig. 5. Zoom of Fig. 4 at low frequencies, showing: (a) absolute value and (b) phase of the tuning coefficient $k(f)$ versus frequency for measurement (markers) and fit to theoretical model (solid lines). The difference between model behavior without $H_{\text {chip }}$ (dashed lines) and measurement indicates the effect of the laser submount interaction.

TABLE III

Fitted Model Parameters For the Best Fit to Measurement as Shown in Figs. 4 And 5

\begin{tabular}{|l|l|l|l|l|l|l|l|l|}
\hline & $k(0){ }^{1}$ & $k_{t h}{ }^{1,2}$ & $\eta_{R}\left(f_{R} / \mathrm{kHz}\right)^{3}$ & $\eta_{Z}\left(f_{Z} / \mathrm{kHz}\right)^{3}$ & $\eta_{D}\left(f_{D} / \mathrm{kHz}\right)^{3}$ & $k_{\text {chip }}{ }^{1,2}$ & $f_{\text {chip }} / \mathrm{Hz}$ & $k_{p l}{ }^{1,2}$ \\
\hline GaAs 763 nm & 287.6 & $289.9(100.8 \%)$ & $0.169(755.6)$ & $0.248(752.6)$ & $0.0492(15.17)$ & $9.2(3.2 \%)$ & 99.4 & $-11.5(-4.0 \%)$ \\
\hline InP 1854 nm & 50.0 & $55.0(109.9 \%)$ & $0.021(41.1)$ & $0.008(373.1)$ & $0.1682(172.91)$ & $1.3(2.7 \%)$ & 28.0 & $-6.3(-12.5 \%)$ \\
\hline InP 2365 nm & 58.4 & $57.9(99.0 \%)$ & $0.034(38.5)$ & $0.133(2357.3)$ & $0.0004(0.38)$ & $2.2(3.8 \%)$ & 38.5 & $-1.6(-2.8 \%)$ \\
\hline GaSb 2330 nm & 49.6 & $49.8(100.5 \%)$ & $0.220(262.6)$ & $0.390(4278.6)$ & $0.0192(1.05)$ & $1.9(3.8 \%)$ & 13.8 & $-2.1(-4.3 \%)$ \\
\hline
\end{tabular}
${ }^{2}$ Strength of individual components. Values given in GHz/mA.
2Relative contribution to overall tuning $k(0)$ given in brackets.
3 The $\eta$ parameters are the relative diffusivities in radial $\left(\eta_{R}\right)$, longitudinal direction $\eta_{Z}$ and the effective diffusivity between the active region
and heat sink $\eta_{D}$.

Except the data for the GaSb-based laser, the measurements were published before [13], [14]. The measurements including the least-squares curve fit to the model (1) is shown in Fig. 4 with a zoom for low frequencies shown in Fig. 5.At the lower end of the frequency scale (see Fig. 5), the effect due to the interaction between submount and laser chip is clearly visible. This effect is present in all investigated VCSELs but with different cutoff frequencies in the range of $5 \mathrm{~Hz}$ (GaSb-based VCSEL) to $100 \mathrm{~Hz}$ (GaAs-based VCSEL) and different relative strength to the overall tuning coefficient, which is attributed to the differ- ent sizes and thicknesses of the VCSEL chips mounted on the submount.

The determined model parameters are summarized in Table III. The $\eta$ values give the normalized "effective" thermal diffusivity in the laser to reproduce the measurements together with the parameters given in Table II. Low $\eta_{R}$ values are caused by a low thermal diffusivity in radial direction in the active region or a larger heat source or mode diameter than assumed. Low $\eta_{Z}$ values are caused by a low thermal diffusivity in longitudinal direction in the active region or a larger lateral heat source or 
mode extension than assumed (as listed in Table II). Finally, low $\eta_{D}$ values indicate a low thermal diffusivity between the active region and the heatsink or a larger effective distance to the heatsink than assumed. Note that in the latter case this is not influenced by the chip mounting technology, because the $\eta$-parameters only describe the intrinsic thermal tuning. A high thermal resistance due to mounting would be described by the $k_{\text {chip }}$ parameter. For the examined VCSEL, the mounting only contributes $2-4 \%$ to the overall thermal resistance.

For time domain computer simulation programs, a zero/pole form of the frequency response is required, whereas the zeros and poles cannot be related directly to physical parameters. In Appendix E, it is shown that the poles must lie on the negative real line and the zeros that best fit the measurement also lie on the negative real line. This proves that the " $N$ time constants" model, which is frequently used in the literature for empirical description of the FM response, is also suited for simulation of VCSELs. Additionally, in Appendix E the zeros and poles for the best fit to the presented measurement data are given.

\section{CONCLUSION}

In this paper, measurements of the FM response (amplitude and phase) for different VCSELs are shown and found to consist of three components (intrinsic thermal tuning, plasma effect, and thermal tuning by laser chip-submount interaction). A physical model for the FM response is developed, which shows good agreement with measurement.

The plasma effect has a significant impact on the FM response (especially the phase shift) starting at frequencies as low as $100 \mathrm{kHz}$. A consequence is that the laser FM tuning behavior cannot be modeled as a minimum phase system, i.e., the FM phase response or time step response cannot be computed via Hilbert transform/Kramers-Kroning methods from only the FM amplitude response. An exception from this is if only the response at frequencies $\ll 100 \mathrm{kHz}$ is of interest. For proper prediction of the wavelength response over a broader frequency range, characterizations of both the amplitude and phase shift are essential.

The third result is that the high-resolution FM phase shift measurements reveal an unexpected peaking of the FM phase shift at low frequencies. This is explained with interaction between the submount and the laser chip. This creates an additional low-intensity tuning effect at low frequencies that contributes another $2 \%-4 \%$ of the overall tuning coefficient.

Fourth, the intrinsic thermal tuning is modeled by a physical laser model with Gaussian-shaped mode and heat source distribution. It reproduces both the slope of $-1 / 2$ in the transition region between cutoff and the start of the plasma effect in the tuning coefficient amplitude as well as the high phase shift of $-90^{\circ}$ for the thermal component. This is achieved by assuming the heat source and mode distribution with a certain thickness. This revealed necessary because investigations showed that models with infinitely thin heat source and mode distribution always create a square root law response with $-45^{\circ}$ phase shift. This improved model allows for a good fit of measured spectra with a low number of parameters.

\section{APPENDIX A}

\section{DEFINITION OF FM AMPLITUdE AND FM PHASE RESPONSE}

For VCSELs, the static current to wavelength tuning behavior can be well described with a quadratic function [27]. For small signal current variations, the tuning behavior can then be considered linear. So for small-signal current excitations, the tunable laser can be considered as a linear time invariant system with respect to its tuning behavior. It is time invariant since the laser has no "memory," i.e., the wavelength response does not depend on the absolute value of time, just the time difference relative to application of the excitation current is relevant. According to systems theory, the response of such systems for a sinusoidal excitation is always sinusoidal with the same frequency

$$
\begin{aligned}
& I(t)=I_{0}+i_{a} \cos (2 \pi f t) \\
& \nu(t)=\nu_{0}-\nu_{a} \cos (2 \pi f t+\phi) .
\end{aligned}
$$

The amplitude of the optical frequency variation $\nu_{a}=\nu_{a}(f)$ and corresponding phase shift $\phi=\phi(f)$ are modulation frequency dependent and form the complex frequency-dependent tuning coefficient

$$
k(f)=\frac{\nu_{a}(f)}{i_{a}} e^{i \phi(f)} .
$$

The negative sign in (14) is added for convenience to obtain a positive value of $k(f)$ at $f=0$ (or $\phi(0)=0$ ). Its absolute value specifies the amplitude of the optical frequency variation under specific current modulation, and the argument of $k(f)$ gives the delay or phase shift between current excitation and wavelength response. If the laser is regarded as a filter with current as input signal and wavelength as output signal, then this complex tuning coefficient $k(f)$ is just the frequency response of the filter. So for a computer simulation knowledge of $k(f)$ is then necessary. The dc tuning coefficient $k(0)$ is real and positive (i.e., $\phi(0)=0$ ) and given by the derivative of the steady-state current to optical frequency relationship $\nu_{0}\left(I_{0}\right)$ with respect to current

$$
k(0)=-\frac{\partial \nu_{0}}{\partial I_{0}} .
$$

The FM response $H(f)$ is the normalized tuning coefficient

$$
H(f)=\frac{k(f)}{k(0)}
$$

and consists of the FM amplitude response $A(f)$ and FM phase response $\phi(f)$

$$
H(f)=A(f) e^{i \phi(f)} .
$$

If the FM response and the dc tuning coefficient is known for all frequencies, then the wavelength response for arbitrary (especially nonsinusoidal) current excitations can be computed.

The FM impulse response and FM step response are equivalent descriptions of the lasers behavior and given by the inverse Fourier transform of the FM response and time integration of 
the FM impulse response, respectively. The FM step response can also be measured as the normalized wavelength response when applying a current step. The FM impulse response plays almost no role in experimental characterization, but is very useful in theoretical investigations both for describing and thermal modeling of the laser.

\section{APPENDIX B}

\section{General Method FOR COMPUTATION OF THE THERMAL PART OF THE FM RESPONSE}

To compute the FM impulse response, the heat equation has to be solved with appropriate boundary conditions and a temporal impulse excitation, i.e.,

$$
c \rho \frac{\mathrm{d} T}{\mathrm{~d} t}-\nabla \cdot(\mathbf{K} \nabla T)=Q \delta(t)
$$

where $c \rho$ is the specific heat capacity times density (unit $\mathrm{J} /\left(\mathrm{m}^{3} \mathrm{~K}\right)$ ), $\mathbf{K}$ the thermal conductivity (unit $\mathrm{W} /(\mathrm{m} \mathrm{K})$ ) matrix and $Q=Q(x, y, z)$ the spatial heat source term (unit $\mathrm{J} / \mathrm{m}^{3}$ ). Equation (19) is in general form where all parameters may depend on space variables $x, y, z$. Here, a constant but nonisotropic thermal conductivity is assumed to account for the nonhomogeneous conductivity of the layered semiconductor material

$$
\mathbf{K}=\left(\begin{array}{ccc}
K_{R} & 0 & 0 \\
0 & K_{R} & 0 \\
0 & 0 & K_{Z}
\end{array}\right)
$$

Boundary conditions of constant temperature like $T(x, y$, $-D)=0$ can be enforced by using the method of images. There the equation without boundary condition is solved with the source term $Q(x, y, z)-Q(x, y, z-2 D)$ instead of $Q$ alone [28].

The solution of (19) with (20) for spatially constant $c \rho$ and spatial impulse heat source $Q(x, y, z)=\delta(x) \delta(y) \delta(z)$ is given by $[28$, Sec. 10.2$]$

$$
T_{p}(x, y, z, t)=\frac{1}{\rho c} G_{\sqrt{2 \kappa_{R} t}}(x) G_{\sqrt{2 \kappa_{R} t}}(y) G_{\sqrt{2 \kappa_{Z} t}}(z) \quad t \geq 0
$$

with the Gaussian function

$$
G_{\sigma}(x)=\frac{1}{\sqrt{2 \pi} \sigma} \exp \left(-\frac{x^{2}}{2 \sigma^{2}}\right)
$$

and $\kappa=K / \rho c$ the thermal diffusivity (unit: $\mathrm{m}^{2} / \mathrm{s}$ ). The general solution of (19) for arbitrary heat source $Q$ is then given by the spatial convolution ${ }^{1}$ of $T_{p}$ with $Q$, i.e.,

$$
T(x, y, z, t)=\left(T_{p}(\cdot, t) * Q\right)(x, y, z) .
$$

The model for the lasers FM response, thus, requires knowledge on the spatial heat source distribution $Q(x, y, z)$ and the light mode profile $M(x, y, z)$. The average temperature with respect

\footnotetext{
${ }^{1}$ Defined as $\left(T_{p}(\cdot, t) * Q\right)(x, y, z)=\iiint T_{p}\left(x-x^{\prime}, y-y^{\prime}, z-z^{\prime}, t\right)$ $Q\left(x^{\prime}, y^{\prime}, z^{\prime}\right) \mathrm{d} x^{\prime} \mathrm{d} y^{\prime} \mathrm{d} z^{\prime}$
}

to the light mode profile $\bar{T}_{g}(t)$ determines the wavelength of the laser, so that the FM impulse response is up to a constant factor compactly written as

$$
\bar{T}_{g}(t)=(T * \widetilde{M})(0,0,0)
$$

with $\widetilde{M}(x, y, z)=M(-x,-y,-z)$ the mirrored mode profile. Summarizing, the FM impulse response is given in the general case by the convolution expression

$$
\bar{T}_{g}(t)=\left(T_{p}(\cdot, t) * Q * \widetilde{M}\right)(0,0,0) .
$$

Equation (25) leads to an important observation. Since the convolution is an associative and commutative operation, the FM response stays the same when the convolution of the mirrored mode profile $\widetilde{M}$ and heat source $Q$ is invariant. This is for example the case when both are Gaussian functions and the mean square of the standard deviations is kept constant (i.e., $\widetilde{M} * Q=\operatorname{const}(x, y, z))$. Or more generally speaking since the convolution operation tends to broaden functions, the device behavior is the same if the light mode is very concentrated but the heat generation very distributed or vice versa. It is the combined width/height (in a mean square sense) of the heat source and light mode distribution that determines the dynamic thermal tuning behavior.

\section{APPENDIX C}

\section{SQUARE ROOT OR PROPORTIONAL BEHAVIOR?}

Most interestingly, two analytic models in the literature for the intrinsic thermal behavior for VCSELs and DFBs [9], [12] predict a square root law behavior for high frequencies, i.e., $H_{\mathrm{th}}(f) \rightarrow 1 / \sqrt{i f}$ for $f \rightarrow \infty$.

Here reasoning is given that the heat equation gives a square root law behavior if the heat source and mode distribution have one dimension less as the mathematical space in which the heat equation is solved. This is for example the case if the heat source and mode have a plane shape in a 3-D space or have a line shape in a 2-D space. From Appendix B, it is known that always the combined thickness of heat source and light mode is relevant. So, a plane heat source and light mode in 3-D space or a line source in 2-D space give a square root behavior. If either the plane heat source or light mode distribution has a nonzero thickness a transition from square root behavior to $1 /(i f)$ behavior will occur at a certain frequency. This second transition frequency is approximately $\kappa /\left(\pi h^{2}\right)$ with (combined) thickness $h$ and thermal diffusivity $\kappa$. The Fourier transform of $1 / \sqrt{t}, t \geq 0$ is given by $1 / \sqrt{2 i f}$, i.e., it has a square root behavior. If, however, $1 / \sqrt{t+\tau}, t \geq 0$ with $\tau>0$ is transformed $e^{2 \pi i f \tau} \operatorname{erfc}(\sqrt{2 \pi i f \tau}) / \sqrt{2 i f}$ is obtained that now has a $1 /(i f)$ behavior for high frequencies. Since the behavior at high frequencies of the Fourier transform is determined by the function behavior around $t=0$, the singularity at $t=0$ of $1 / \sqrt{t}$ seems to be essential for causing the square root behavior. Further investigations of solutions of heat equations of different dimensions showed that this $1 / \sqrt{t}$ term only exists if the heat source and light mode have a lower dimension as the space in which the heat equation is solved. 
TABLE IV

FitTEd POLES AND ZEROS FOR EMPIRICAL MODEL (26)

\begin{tabular}{|l|l|l|l|l|l|l|l|}
\hline & $k(0)^{1}$ & $k_{t h}{ }^{1,2}$ & "poles" $f_{k}{ }^{\prime} \mathrm{kHz}$ & "zeros" $-z_{k} /(2 \pi) / \mathrm{kHz}$ & $k_{\text {chip }}{ }^{1,2}$ & $f_{\text {chip }} / \mathrm{Hz}$ & $k_{p l}{ }^{1,2}$ \\
\hline GaAs 763 nm & 287.7 & $288.5(100.3 \%)$ & $15.86,127.40,856.15$ & $18.27,239.24$ & $9.4(3.3 \%)$ & 103.3 & $-10.2(3.6 \%)$ \\
\hline InP 1854 nm & 50.0 & $54.9(109.9 \%)$ & $22.47,91.58,377.70$ & $25.54,169.83$ & $1.3(2.6 \%)$ & 31.5 & $-6.2(12.5 \%)$ \\
\hline InP 2365 nm & 58.4 & $56.5(96.6 \%)$ & $1.85,13.64,107.39,648.57$ & $2.39,25.36,308.07$ & $3.5(6.0 \%)$ & 57.5 & $-1.5(2.6 \%)$ \\
\hline GaSb 2330 nm & 49.7 & $49.4(99.2 \%)$ & $0.80,22.98,202.02,1077.32$ & $0.83,35.41,449.30$ & $1.8(3.6 \%)$ & 11.0 & $-1.4(2.9 \%)$ \\
\hline
\end{tabular}

${ }^{1}$ All values given in $\mathrm{GHz} / \mathrm{mA}$.

${ }^{2}$ Relative contribution to overall tuning $k(0)$ also given in brackets.

\section{APPENDIX D}

\section{COMPUTATION OF (7) AND (11)}

Efficient numerical evaluation of (7), where the desired frequency points are distributed over several orders of magnitude, can be carried out in the following manner: the integral is of the form $\int_{0}^{\infty} f(t) e^{-2 \pi i f t} \mathrm{~d} t$ with $f(t)$ an nonoscillatory and falling function that is now assumed to be evaluated at $N$ discrete points $t_{1}, \ldots, t_{N}$. Then $f(t)$ is approximated by the function that linearly interpolates the discrete points $f\left(t_{i}\right)$ and is zero for $t>t_{N}$. The Fourier transform of a piecewise linear function is easily obtained analytically, which leaves the question how to chose the samples $t_{i}$ to achieve a certain accuracy at the desired frequency points. The behavior of the Fourier transform at high frequencies is mainly determined by the functions behavior around $t=0$, while the behavior at $\mathrm{dc}(f \approx 0)$ depends on all function values of $f(t)$. If $H_{\mathrm{th}}(f)$ is to be computed at frequency points over a wide range of magnitudes, sampling of $f(t)$ over a wide range and dense sampling around $t=0$ is required. It turned out that logarithmically spacing from $t_{2}=10^{-15}$ to $t_{N}=10^{2}$ with $t_{1}=0$ and $N=500$ points gives very good results with low computational effort.

In a practical measurement, only discrete points $A\left(f_{i}\right)$ are available for computation of the Hilbert transform (11). To compute (11), it is necessary to place some assumptions on the behavior of the function between the points $f_{i}$ and outside the measured frequency interval $\left[f_{\min }, f_{\max }\right]$. In this study, $\log A(f)$ is assumed to be linearly interpolating between two measurements and to extend linearly to $f \rightarrow \infty$ with a slope determined from the last few points (linear extrapolation). The analytical value of the integral (11) for a symmetric and piecewise linear function is easily obtained. This method is particularly suited for irregularly spaced frequency points $f_{i}$ that span several orders of magnitude.

\section{APPENDIX E}

\section{EMPIRICAL FM RESPONSE MODEL}

For a pure computer simulation of a system containing a tunable laser the FM response must be present in a rational form. ${ }^{2}$ A rational frequency response always corresponds to a system that is described with ordinary differential equation in the time domain. The first-order low-pass (one "time constant") or the

\footnotetext{
${ }^{2}$ This means that the frequency response it is a quotient of two polynomials with real coefficients in the variable $s=2 \pi i f$.
}

" $N$ time constants" model is of such a rational form. Time domain simulation programs like "SPICE" or "Simulink" require models to be rational. In such a model, however, the obtained parameters (zeros and poles) cannot be related to real physical quantities inside the laser device. For pure mathematical description of measured data, e.g., for computer simulation of a laser system, this approach can be powerful, since with a certain number of time constants arbitrary FM responses can be fitted. For modeling of the intrinsic thermal tuning, all poles $p_{k}$ have to lie on the negative real line. They have to be real, because a thermal defined system does not describe oscillations at an impulse excitation. A negative real part is required for the system to be stable. The zeros also must have negative real part to describe a minimum phase system. Since the heat equation only contains the first-order time derivative an asymptotic slope between 0 and -1 or $\left|H_{\mathrm{th}}(f)\right| \rightarrow 1 / f$ for high frequencies is expected. The only meaningful selection for the numerator degree is, thus, one minus the denominator degree (asymptotic $1 /($ if $)$ behavior). Thus, the following model is used:

$$
H_{\mathrm{th}}(f)=\frac{\prod_{k=1}^{N-1}\left(1-2 \pi i f / z_{k}\right)}{\prod_{k=1}^{N}\left(1+i f / f_{k}\right)} .
$$

The poles are $p_{k}=-2 \pi f_{k}$ and $z_{k}$ the (possibly complex) zeros. If a zero is complex, then the conjugate complex zero must be a zero as well, so that using complex zeros does not increase the degree of freedom of the model (which is $2 N-1$ ). Note that also multiple zeros or poles can be present (i.e., zeros/poles of higher order).

In the case of single poles and real zeros, the model (26) can be simplified to the " $N$ time constants" model [11], [29]

$$
H_{\mathrm{th}}(f)=\sum_{k=1}^{N} a_{k} \frac{1}{1+i f / f_{k}}
$$

with positive values $a_{k}$ and distinct characteristic frequencies $f_{k}$. For $a_{k}>0$, this even always describes a minimum phase system with real zeros. For $a_{k}<0$, this is not necessarily the case, but also complex zeros can be described. Note that this is not a fully general model, since it cannot describe multiple poles. This is even so if some $f_{k}$ are chosen to be equal. This can be easily seen if the partial fraction decomposition of (26) is computed: in the case of multiple poles also terms $1 /(1+$ if $\left./ f_{k}\right)^{r_{k}}$ with $r_{k}>1$ would have to be present in (27). For the measurement data of the VCSELs studied in this paper, the best fit to model (26) did not produce complex zeros or poles with multiplicity greater than one. This empirically proves that for the 
specific measurement data the " $N$ time constants" model (27) is indeed suitable. The necessary order was between $N=3$ or $N=4$. Interestingly, the necessary order can be estimated from the fit itself. If $N$ is chosen to be "too large" in the beginning, then the fit will produce a zero and a pole that are lying very close by. So their contribution in (26) will nearly cancel out, which indicates that $N$ can be chosen lower. The fitted zeros and poles are shown in Table IV.

\section{REFERENCES}

[1] P. V. Mena, J. J. Morikuni, S.-M. Kang, A. V. Harton, and K. W. Wyatt, "A comprehensive circuit-level model of vertical-cavity surface-emitting lasers," J. Lightwave Technol., vol. 17, no. 12, pp. 2612-2632, Dec. 1999.

[2] R. D. Esman and D. L. Rode, "Semiconductor-laser thermal time constant," J. Appl. Phys., vol. 59, pp. 407-409, Jan. 1986.

[3] P. K. L. Chan, K. P. Pipe, Z. Mi, J. Yang, P. Bhattacharya, and D. Lüerßen, "Thermal relaxation time and heat distribution in pulsed InGaAs quantum dot lasers," Appl. Phys. Lett., vol. 89, no. 1, pp. 011110-1-011110-3, 2006.

[4] H. I. Abdelkader, H. H. Hausien, and J. D. Martin, "Temperature rise and thermal rise-time measurements of a semiconductor laser diode," Rev. Sci. Instrum., vol. 63, pp. 2004-2007, Mar. 1992.

[5] K. Petermann, Laser Diode Modulation and Noise. Norwell, MA: Kluwer, 1988

[6] M. Lackner, G. Totschnig, F. Winter, M. Ortsiefer, M.-C. Amann, R. Shau, and J. Rosskopf, "Demonstration of methane spectroscopy using a verticalcavity surface-emitting laser at $1.68 \mu \mathrm{m}$ with up to $5 \mathrm{MHz}$ repetition rate," Meas. Sci. Technol., vol. 14, pp. 101-106, 2003.

[7] J. Chen, A. Hangauer, R. Strzoda, and M.-C. Amann, "Experimental characterization of the frequency modulation behavior of vertical cavity surface emitting lasers," Appl. Phys. Lett., vol. 91, no. 14, pp. 141105-1141105-3, Oct. 2007.

[8] M.-C. Amann and J. Buus, Tunable Laser Diodes. Norwood, MA: Artech House, 1998.

[9] G. Pandian and S. Dilwali, "On the thermal FM response of a semiconductor laser diode," IEEE Photon. Technol. Lett., vol. 4, no. 2, pp. 130-133, Feb. 1992

[10] G. Hasnain, K. Tai, L. Yang, Y. H. Wang, R. J. Fischer, J. D. Wynn, B. Weir, N. K. Dutta, and A. Y. Cho, "Performance of gain-guided surface emitting lasers with semiconductor distributed Bragg reflectors," IEEE J. Quantum Electron., vol. 27, no. 6, pp. 1377-1385, Jun. 1991.

[11] H. Shalom, A. Zadok, M. Tur, P. J. Legg, W. D. Cornwell, and I. Andonovic, "On the various time constants of wavelength changes of a DFB laser under direct modulation," IEEE J. Quantum Electron., vol. 34, no. 10 , pp. 1816-1822, Oct. 1998.

[12] J. Chen, A. Hangauer, and M.-C. Amann, "Simplified model of the dynamic thermal tuning behavior of vertical-cavity surface-emitting laser," IEEE Photon. Technol. Lett., vol. 20, no. 13, pp. 1082-1084, Jul. 2008.

[13] A. Hangauer, J. Chen, R. Strzoda, and M. Amann, "High-speed tuning in vertical-cavity surface-emitting lasers," presented at the Eur. Conf. Lasers Electro-Opt., Munich, Germany, Jun. 2009, p. CB13.5.

[14] A. Hangauer, J. Chen, and M.-C. Amann, "Comparison of plasma-effect in different InP-based VCSELs," presented at the Conf. Lasers Electro-Opt. (CLEO), May 2010, San Jose, CA, p. CMO4.

[15] G. Böhm, M. Grau, O. Dier, K. Windhorn, E. Rönneberg, J. Rosskopf, R. Shau, R. Meyer, M. Ortsiefer, and M.-C. Amann, "Growth of InAscontaining quantum wells for InP-based VCSELs emitting at $2.3 \mu \mathrm{m}$," $J$. Cryst. Growth, vol. 301, pp. 941-944, Apr. 2007.

[16] M. Ortsiefer, G. Böhm, M. Grau, K. Windhorn, E. Rönneberg, J. Rosskopf, R. Shau, O. Dier, and M.-C. Amann, "Electrically pumped room temperature CW VCSELs with $2.3 \mu \mathrm{m}$ emission wavelength," Electron. Lett., vol. 42, no. 11, pp. 640-641, May 25, 2006.

[17] H.E. Li and K. Iga, Eds., Vertical-Cavity Surface-Emitting Laser Devices, 1st ed.. New York: Springer-Verlag, 2003.

[18] J. Piprek, D. I. Babic, and J. E. Bowers, "Simulation and analysis of $1.55 \mu \mathrm{m}$ double-fused vertical-cavity lasers," J. Appl. Phys., vol. 81, no. 8, pp. 3382-3390, Apr. 1997.

[19] C. Henry, "Theory of the linewidth of semiconductor lasers," IEEE J. Quantum Electron., vol. QE-18, no. 2, pp. 259-264, Feb. 1982.

[20] J. Huang and L. W. Casperson, "Gain and saturation in semiconductor lasers," Opt. Quantum Electron., vol. 25, no. 6, pp. 369-390, Jun. 1993.
[21] J. Gustavsson, A. Haglund, J. Bengtsson, P. Modh, and A. Larsson, "Dynamic behavior of fundamental-mode stabilized VCSELs using a shallow surface relief," IEEE J. Quantum Electron., vol. 40, no. 6, pp. 607-619, Jun. 2004.

[22] A. V. Oppenheim, R. W. Schafer, and J. R. Buck, Discrete-Time Signal Processing, 2nd ed. Upper Saddle River, NJ: Prentice-Hall, 1999.

[23] M. Grabherr, D. Wiedenmann, R. Jaeger, and R. King, "Fabrication and performance of tunable single-mode VCSELs emitting in the 750 to 1000 nm range," Proc. SPIE, vol. 5737, pp. 120-128, Mar. 2005.

[24] M.-C. Amann and M. Ortsiefer, "Long-wavelength $(\lambda \geq 1.3 \mu \mathrm{m})$ InGaAlAs-InP vertical-cavity surface-emitting lasers for applications in optical communication and sensing," Phys. Status Solidi A, vol. 203, pp. 3538-3544, Aug. 2006.

[25] A. Bachmann, K. Kashani-Shirazi, T. Lim, O. Dier, C. Lauer, and M.-C. Amann, "Single-mode continuous wave operation of electrically pumped $2.25 \mu \mathrm{m} \mathrm{GaSb}$-based VCSEL," presented at the 20th Int. Conf. Indium Phosphide Related Mater., Versailles, France, 2008.

[26] A. Bachmann, T. Lim, K. Kashani-Shirazi, O. Dier, C. Lauer, and M.-C. Amann, "Continuous-wave operation of electrically pumped GaSb-based vertical cavity surface emitting laser at $2.3 \mu \mathrm{m}$," Electron. Lett., vol. 44, no. 3, pp. 202-203, Jan. 2008.

[27] A. Lytkine, W. Jäger, and J. Tulip, "Frequency tuning of long-wavelength VCSELs," Spectro. Acta Part A: Mol. Biomol. Spectrosc., vol. 63, no. 5, pp. 940-946, 2006 (5th Int. Conf. Tunable Diode Laser Spectrosc., 2005).

[28] H. Carslaw and J. Jaeger, Conduction of Heat in Solids. Oxford, U.K.: Clarendon, 1990

[29] I. Roudas, Y. Jaouen, J. Prado, R. Vallet, and P. Gallion, "Accurate model of the semiconductor laser nonuniform FM response for the study of coherent optical systems," IEEE Photon. Technol. Lett., vol. 6, no. 11, pp. 1389-1391, Nov. 1994.

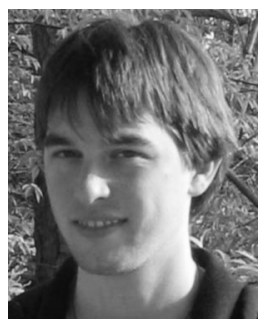

Andreas Hangauer (M'08) was born in Aachen, Germany. He received the Diploma in electrical engineering from the University of Karlsruhe, Karlsruhe, Germany, in Spring 2006, and the Mathematics Diploma degree from the Technical University of Munich, Munich, Germany, in 2010. He is currently working towards the Doctorate degree at the Technical University of Munich in close collaboration with the Siemens Corporate Technology laboratories in Munich.

$\mathrm{He}$ is working on optical gas sensing with laser spectroscopy using vertical-cavity surface-emitting lasers and investigates advanced detection schemes for optical gas sensing with simulation of laser optical gas sensors and modeling of their components.

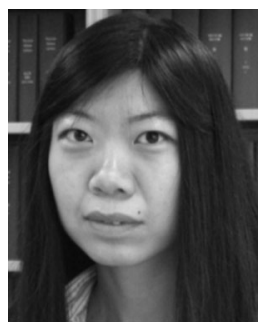

Jia Chen (M'08) received the Diploma degree from the University of Karlsruhe, (now the Karlsruhe Institute of Technology) Karlsruhe, Germany, in Spring 2006, and the Doctorate degree from the Technical University of Munich in January 2011, both in electrical engineering. She did her doctoral thesis at the Walter Schottky Institute in close cooperation with Siemens AG for compact optical gas sensing with vertical-cavity surface-emitting lasers and hollow capillary fibers.

Her research interests include modeling of surfaceemitting lasers, in-fiber-based gas sensing, and new methodologies for signal evaluation. 


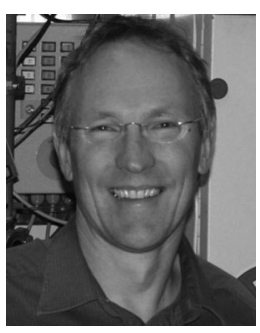

Rainer Strzoda born in 1957 in Bavaria, Germany. He received the Diploma in physics from the Technical University of Munich, Munich, Germany, in 1986.

Joining the Siemens R\&D Labs in Munich he first developed epitaxy-processes for electro-optical components used in fiber communication systems and later designed and characterized semiconductor optical amplifiers. Moving to the Sensors and Actuator group in 1995, he is working on tunable diode laser spectroscopy (TDLS) in a variety of projects, with a clear focus on low cost designs, which enables TDLS to break into the mid and low cost market segments.

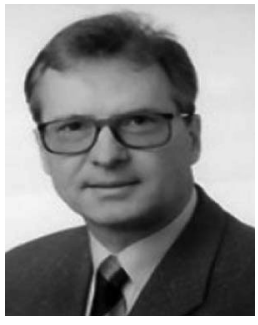

Markus-Christian Amann (A'88-SM'91-F'07) was born in Singen/Hohentwiel, Germany, in 1950. $\mathrm{He}$ received the Diploma degree in electrical engineering and the Dr.-Ing. degree from the Technical University of Munich, Munich, Germany, in 1976 and 1981, respectively.

During his thesis work, he studied superluminescent diodes and low-threshold laser diodes, and developed the AlGaAs-GaAs metal-clad ridge-waveguide laser. From 1981 to 1994, he was with the Corporate Research Laboratories, Siemens AG, Munich, where he was engaged in research on long-wavelength InGaAsP-InP laser diodes. In 1990, he became a Deputy Director for the research on laser diodes and integrated optoelectronic devices. On February 1994, he joined the Department of Electrical Engineering at the University of Kassel as a Full professor for "Technical Electronics" establishing a working group for III/V semiconductor electronics and optoelectronics. Since November 1997, he holds the Chair of Semiconductor Technology at the Walter Schottky Institute, Technical University of Munich, where he is currently engaged in research on tunable laser diodes for the near-IR, quantum cascade lasers, long-wavelength vertical-cavity laser diodes, and laser diode applications. He has authored or coauthored some 400 papers and talks (including some 50 invited) on semiconductor optoelectronics in scientific journals, conference proceedings. He has coauthored two books.

Prof. Amann is a member of the German Informationstechnische Gesellschaft (ITG), and a Fellow of the IEEE Lasers and Electro-Optics Society. He has served on numerous conference committees such as the IEEE Semiconductor Laser Conferences, the Indium Phosphide and Related Materials Conferences, and the Conferences on Lasers and Electro-Optics. 